\title{
Capecitabine in Combination with Endocrine Therapy as Maintenance Therapy after Bevacizumab Plus Paclitaxel Induction Therapy for Hormone Receptor-Positive, HER2-Negative Metastatic Breast Cancer: KBCSG-TR1214
}

\author{
Norikazu Masuda ${ }^{1, *,+} \oplus$, Tetsuhiro Yoshinami ${ }^{2,3, \dagger}$, Masahiko Ikeda ${ }^{4}$, Makiko Mizutani ${ }^{1}$, Miki Yamaguchi ${ }^{5}$, \\ Yoshifumi Komoike ${ }^{6}{ }^{\circledR}$, Tsutomu Takashima ${ }^{7}{ }^{\mathbb{D}}$, Katsuhide Yoshidome ${ }^{8}{ }^{\mathbb{D}}$, Junji Tsurutani ${ }^{9}{ }^{\circledR}$, \\ Mitsuhiko Iwamoto ${ }^{10}$, Fumie Fujisawa ${ }^{2}{ }^{\mathbb{D}}$, Hiroyuki Yasojima ${ }^{1}$, Jun Yamamura ${ }^{11}{ }^{\mathbb{D}}$, Hirotaka Morishima ${ }^{12}$, \\ Fuminori Aki ${ }^{13}$, Tomomi Yamada ${ }^{14}$, Satoshi Morita ${ }^{15}$ and Takahiro Nakayama ${ }^{16}$ (i)
}

check for

updates

Citation: Masuda, N.; Yoshinami, T. Ikeda, M.; Mizutani, M.;

Yamaguchi, M.; Komoike, Y.;

Takashima, T.; Yoshidome, K.;

Tsurutani, J.; Iwamoto, M.; et al. Capecitabine in Combination with Endocrine Therapy as Maintenance Therapy after Bevacizumab Plus Paclitaxel Induction Therapy for Hormone Receptor-Positive, HER2-Negative Metastatic Breast Cancer: KBCSG-TR1214. Cancers 2021, 13, 4399. https://doi.org/10.3390/ cancers13174399

Academic Editor: Rupert Bartsch

Received: 31 May 2021

Accepted: 27 August 2021

Published: 31 August 2021

Publisher's Note: MDPI stays neutral with regard to jurisdictional claims in published maps and institutional affiliations.

Copyright: (C) 2021 by the authors Licensee MDPI, Basel, Switzerland. This article is an open access article distributed under the terms and conditions of the Creative Commons Attribution (CC BY) license (https:// creativecommons.org/licenses/by/ $4.0 /)$.
1 Department of Surgery, Breast Oncology, National Hospital Organization Osaka National Hospital, 2-1-14 Hoenzaka, Chuou-ku, Osaka-shi 540-0006, Osaka, Japan; mizutani.makiko.ay@mail.hosp.go.jp (M.M.); yasojima.hiroyuki.wt@mail.hosp.go.jp (H.Y.)

2 Department of Medical Oncology, Osaka International Cancer Institute, 3-1-69 Otemae, Chuo-ku, Osaka-shi 541-8567, Osaka, Japan; yosinami-te@onsurg.med.osaka-u.ac.jp (T.Y.); fujisawa-fu@mc.pref.osaka.jp (F.F.)

3 Breast and Endocrine Surgery, Graduate School of Medicine, Osaka University, 2-2-E10 Yamadaoka, Suita-shi 565-0871, Osaka, Japan

4 Department of Breast and Thyroid Surgery, Fukuyama City Hospital, 5-23-1 Zao-cho, Fukuyama-shi 721-8511, Hiroshima, Japan; masahikoikeda@city.fukuyama.hiroshima.jp

5 Department of Breast Surgery, JCHO Kurume General Hospital, 21 Kushiharamachi, Kurume-shi 830-0013, Fukuoka, Japan; yamaguchi-miki@kurume.jcho.go.jp

6 Department of Medicine, Kindai University Hospital, 377-2 Ohnohigashi, Osaka-Sayama-shi 589-8511, Osaka, Japan; komoike@med.kindai.ac.jp

7 Department of Breast and Endocrine Surgery, Graduate School of Medicine, Osaka City University, 1-4-3 Asahi-machi, Abeno-ku, Osaka-shi 545-8585, Osaka, Japan; m1158973@med.osaka-cu.ac.jp

8 Department of Breast and Endocrine Surgery, Osaka Police Hospital, 10-31 Kitayama-cho, Tennouji-ku, Osaka-shi 543-0035, Osaka, Japan; yoshidome-k@umin.ac.jp

9 Advanced Cancer Translation Research Institute, Showa University, 1-5-8 Hatanodai, Shinagawa-ku 142-8555, Tokyo, Japan; tsurutaj@med.showa-u.ac.jp

10 Breast and Endocrine Surgery, Osaka Medical College, 2-7 Daigakumachi, Takatsuki-shi 569-8686, Osaka, Japan; sur067@poh.osaka-med.ac.jp

11 Department of Surgery, Sakai City Medical Center, 1-1-1 Ebaraji-cho, Nishi-ku, Sakai-shi 593-8304, Osaka, Japan; june10th@zeus.eonet.ne.jp

12 Department of Breast Surgery, Osaka Rosai Hospital, 1179-3 Nagasone-cho, Kitaku, Sakai-shi 591-8025, Osaka, Japan; morisima@bd5.so-net.ne.jp

13 Ito Surgical Mammary Gland Clinic, 12-10 Fudaba, Kochi-shi 781-0085, Kochi, Japan; suito@basil.ocn.ne.jp

14 Department of Medical Innovation, Osaka University Hospital, 2-2, Yamadaoka, Suita-shi 565-0871, Osaka, Japan; tomomi.yamada@dmi.med.osaka-u.ac.jp

15 Department of Biomedical Statistics and Bioinformatics, Kyoto University Graduate School of Medicine, 54 Kawahara-cho, Shogoin, Sakyo-ku, Kyoto-shi 606-8507, Kyoto, Japan; smorita@kuhp.kyoto-u.ac.jp

16 Department of Breast and Endocrine Surgery, Osaka International Cancer Institute, 3-1-69 Otemae, Chuo-ku, Osaka-shi 541-8567, Osaka, Japan; taqnakayama@gmail.com

* Correspondence: nmasuda@alpha.ocn.ne.jp; Tel.: +81-6-6942-1331

+ These two authors contributed equally to this work.

Simple Summary: To investigate a possible treatment strategy for hormone receptor (HR)-positive, HER2-negative advanced and/or metastatic breast cancer (AMBC), we investigated the clinical usefulness of adding capecitabine to maintenance endocrine therapy after induction chemotherapy and the efficacy of reinduction chemotherapy. Patients who had received bevacizumab-paclitaxel induction therapy and did not have progressive disease were randomized to receive maintenance therapy with endocrine therapy alone (group E; $n=46$ ) or endocrine therapy plus capecitabine (group EC; $n=44$ ). The median progression-free survival (PFS) under maintenance therapy (primary endpoint) was significantly longer in group EC than in group E (11.1 vs. 4.3 months; hazard ratio, $0.53 ; p<0.01)$. At 24 months from the induction therapy start, the overall survival (OS) rate was 
significantly higher in group EC than in group E ( $83.5 \%$ vs. $62.3 \% ; p=0.02)$. Therefore, the addition of capecitabine to maintenance endocrine therapy may be a beneficial option after induction chemotherapy for HR-positive, HER2-negative AMBC patients.

Abstract: Optimal treatment strategies for hormone receptor (HR)-positive, HER2-negative advanced and/or metastatic breast cancer (AMBC) remain uncertain. We investigated the clinical usefulness of adding capecitabine to maintenance endocrine therapy after induction chemotherapy and the efficacy of reinduction chemotherapy. Patients who had received bevacizumab-paclitaxel induction therapy and did not have progressive disease (PD) were randomized to maintenance therapy with endocrine therapy alone (group E) or endocrine plus capecitabine (1657 mg/m² / day on days 1-21, $\mathrm{q} 4 \mathrm{w}$ ) (group EC). In case of PD after maintenance therapy, patients received bevacizumab-paclitaxel reinduction therapy. Ninety patients were randomized. The median progression-free survival (PFS) under maintenance therapy (primary endpoint) was significantly longer in group EC (11.1 \{95\% CI, 8.0-11.8 months) than in group E (4.3 $\{3.6-6.0\}$ months) (hazard ratio, $0.53 ; p<0.01)$. At 24 months from the induction therapy start, the overall survival (OS) was significantly longer in group EC than in group $\mathrm{E}$ (hazard ratio, $0.41 ; p=0.046$ ). No difference was found in the time to failure of strategy (13.9 and 16.6 months in groups E and EC, respectively). Increased capecitabine-associated toxicities in group EC were tolerable. Addition of capecitabine to maintenance endocrine therapy may be a beneficial option after induction chemotherapy for HR-positive, HER2-negative AMBC patients.

Keywords: advanced and metastatic breast cancer; bevacizumab; capecitabine; endocrine therapy; hormone receptor positive; HER2-negative breast cancer; maintenance therapy; randomized trial

\section{Introduction}

The aim of treatment for advanced and/or metastatic breast cancer (AMBC) is to prolong patients' survival and improve their quality of life (QOL) by controlling disease symptoms. For hormone receptor (HR)-positive, human epidermal growth factor receptor 2 (HER2)-negative breast cancer, which is the most common subtype of advanced breast cancer, endocrine therapy, as many regimens as possible, is recommended [1]. Moreover, adding molecular targeted drugs, such as the recently developed cyclin-dependent kinase 4 and 6 (CDK4/6) inhibitors, to endocrine therapy has become recent standard first- or second-line treatment [2-7]. However, as resistance to these drugs may develop, or in case of life-threatening conditions, such as visceral involvement, chemotherapy is the treatment of choice. In the hope of a rapid response, bevacizumab in combination with an anticancer drug is considered.

Bevacizumab has been shown to significantly increase progression-free survival (PFS) and improve the response rate when combined with docetaxel [8,9] or paclitaxel $[10,11]$ in AMBC patients. In Japan, weekly paclitaxel plus bevacizumab is widely used as one of the standard therapy regimens for HER2-negative metastatic breast cancer [12]. However, adverse events (AEs) of these drugs, such as peripheral neuropathy, hypertension, and proteinuria, are a concern as they may reduce patients' QOL over the long term. In general, a longer duration of first-line chemotherapy is associated with prolonged PFS and overall survival (OS) [13], so intensive treatment tends to be continued until disease progression or intolerable toxicity in daily clinical practice, while AEs need to be minimized to maintain patients' QOL.

Maintenance therapy may be one way to achieve this balance. At the Fifth International Consensus Conference for Advanced Breast Cancer, experts agreed that in cases of HR-positive, HER2-negative AMBC in which induction chemotherapy has been effective, endocrine maintenance therapy is a reasonable treatment option [14,15]. Induction therapy followed by maintenance therapy is expected to improve survival and QOL in AMBC.

Furthermore, capecitabine is expected to be beneficial for the treatment of AMBC. The IMELDA [16] and CREATE-X [17] trials have shown the efficacy of capecitabine against 
high-risk breast cancer in cases of non-cross-resistance with intravenous chemotherapeutic agents, with good compliance. For HR-positive, HER2-negative AMBC, the usefulness of the addition of capecitabine to bevacizumab as maintenance therapy has been suggested [16,18]; however, the current evidence regarding effective maintenance therapy is limited and needs to be explored.

Additionally, the strategy after maintenance therapy is also important in view of how effectively anticancer drugs can be used in the treatment of AMBC. A Japanese study has shown that reinduction therapy with bevacizumab and paclitaxel, following temporal discontinuation of paclitaxel due to its AEs, is safe and effective [19].

Based on these findings, we conducted the present multicenter randomized phase 2 trial (KBCSG-TR1214) with the following aims: (1) to investigate the clinical usefulness of the addition of capecitabine to endocrine therapy, compared with endocrine therapy alone, as maintenance therapy after induction chemotherapy with bevacizumab and paclitaxel and (2) to evaluate the efficacy of bevacizumab-paclitaxel reinduction therapy after failure of maintenance therapy in patients with HR-positive, HER2-negative AMBC.

\section{Results}

\subsection{Patient Characteristics}

A total of 116 patients were enrolled between August 2012 and April 2016 and received induction therapy with bevacizumab and paclitaxel. Figure 1 shows the patient flow. Of the 116 patients enrolled, 26 did not proceed to randomization, mainly due to progressive disease (PD). The remaining 90 patients were randomized to endocrine-only therapy (group E, $n=46$ ) or endocrine therapy plus capecitabine (group EC, $n=44$ ). Allocation to treatment groups ended in October 2016.

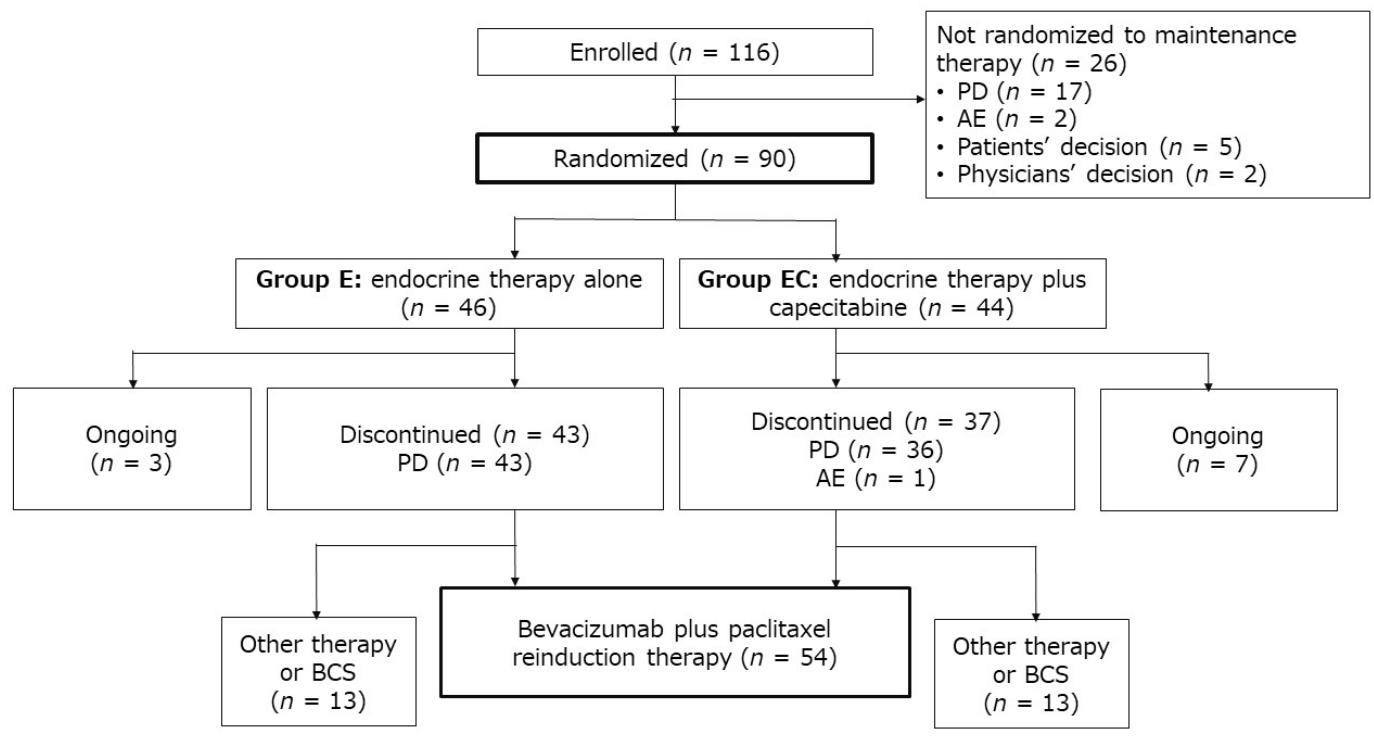

Figure 1. Patient disposition. AE, adverse event; BCS, breast-conserving surgery; E, endocrine therapy alone; EC, endocrine therapy plus capecitabine; PD, progressive disease.

Patients in the two groups were well balanced in terms of baseline characteristics (Table 1). The median age was 59.8 years (range, 41.5-75.8 years) in group E and 59.9 years (range, 34.5-81.0 years) in group EC. In each group, just over a fifth of the patients were premenopausal. Of the 90 patients randomized, $85(94.4 \%)$ had a measurable lesion, and 81 $(90.0 \%)$ had received no prior chemotherapy for AMBC and therefore received bevacizumab plus paclitaxel as first-line chemotherapy. Most $(75 / 90,83.3 \%)$ had completed 6 cycles of bevacizumab-paclitaxel induction therapy, and the remaining 15 (16.7\%) had received 4 or 5 cycles (data not shown). 
Table 1. Baseline characteristics.

\begin{tabular}{|c|c|c|c|c|}
\hline Characteristic & $\begin{array}{l}\text { Enrolled } \\
(n=116)\end{array}$ & $\begin{array}{l}\text { Randomized } \\
\quad(n=90)\end{array}$ & $\begin{array}{l}\text { Group E } \\
(n=46)\end{array}$ & $\begin{array}{c}\text { Group EC } \\
(n=44)\end{array}$ \\
\hline $\begin{array}{c}\text { Median Age, Years } \\
\text { (Range) }\end{array}$ & $59.8(31.5-81.0)$ & $59.8(34.5-81.0)$ & $59.8(41.5-75.8)$ & $59.9(34.5-81.0)$ \\
\hline \multicolumn{5}{|l|}{ Menopausal } \\
\hline Premenopausal & $26(22.4)$ & $20(22.2)$ & $10(21.7)$ & $10(22.7)$ \\
\hline $\begin{array}{c}\text { Postmenopausal } \\
\text { ECOG PS }\end{array}$ & $90(77.6)$ & $70(77.8)$ & $36(78.3)$ & $34(77.3)$ \\
\hline 0 & 83 (71.6) & $64(71.1)$ & $33(71.7)$ & $31(70.5)$ \\
\hline 1 & $32(27.6)$ & $25(27.8)$ & $13(28.3)$ & $12(27.3)$ \\
\hline 2 & $1(0.9)$ & $1(1.1)$ & 0 & $1(02.3)$ \\
\hline \multicolumn{5}{|l|}{$\underset{\mathrm{a}}{\operatorname{Measurable~Lesion}}$} \\
\hline Yes & $111(95.7)$ & $85(94.4)$ & $44(95.7)$ & $41(93.2)$ \\
\hline No & $5(4.3)$ & $5(5.6)$ & $2(4.3)$ & $3(6.8)$ \\
\hline \multicolumn{5}{|l|}{ Previous } \\
\hline \multicolumn{5}{|l|}{ Endocrine } \\
\hline \multicolumn{5}{|l|}{ Therapies } \\
\hline \multicolumn{5}{|l|}{ with/without } \\
\hline \multicolumn{5}{|l|}{$\begin{array}{l}\text { Targeted Therapy } \\
\text { for AMBC }\end{array}$} \\
\hline 0 & $42(36.2)$ & $35(38.9)$ & $18(39.1)$ & $17(38.6)$ \\
\hline 1 Regimen & $26(22.4)$ & $13(14.4)$ & $7(15.2)$ & $6(13.6)$ \\
\hline 2 Regimens & $19(16.4)$ & $16(17.8)$ & $8(17.4)$ & $8(18.2)$ \\
\hline$\geq 3$ Regimens & $29(25.0)$ & $26(28.9)$ & $13(28.3)$ & $13(29.5)$ \\
\hline $\begin{array}{l}\text { Exemestane + } \\
\text { mTOR Inhibitor } \\
\text { Previous }\end{array}$ & $8(6.9)$ & $7(7.8)$ & $4(8.7)$ & $3(6.8)$ \\
\hline \multicolumn{5}{|l|}{$\begin{array}{l}\text { Chemotherapy for } \\
\text { AMBC }\end{array}$} \\
\hline 0 & $102(87.9)$ & $81(90.0)$ & $39(84.8)$ & $42(95.5)$ \\
\hline 1 Regimen & $14(12.1)$ & $9(10.0)$ & $7(15.2)$ & $2(4.5)$ \\
\hline \multicolumn{5}{|l|}{ Histological Grade } \\
\hline 1 & $19(16.4)$ & $15(16.7)$ & $8(17.4)$ & 7 (15.9) \\
\hline 2 & $41(35.3)$ & $32(35.6)$ & $17(37.0)$ & $15(34.1)$ \\
\hline 3 & $23(19.8)$ & $15(16.7)$ & $6(13.0)$ & $9(20.5)$ \\
\hline Unknown & $33(28.4)$ & $28(31.1)$ & $15(32.6)$ & $13(29.5)$ \\
\hline Hormone & & & & \\
\hline \multicolumn{5}{|l|}{ Receptor Status } \\
\hline $\mathrm{ER}+\mathrm{PgR}+$ & $96(82.8)$ & $75(83.3)$ & $38(82.6)$ & $37(84.1)$ \\
\hline $\mathrm{ER}+\mathrm{PgR}-$ & $18(15.5)$ & $13(14.4)$ & $7(15.2)$ & $6(13.6)$ \\
\hline $\begin{array}{l}\text { ER }+ \text { PgR } \\
\text { Unknown }\end{array}$ & $2(1.7)$ & $2(2.2)$ & $1(2.2)$ & $1(2.3)$ \\
\hline \multicolumn{5}{|l|}{ HER2 Status (IHC) } \\
\hline 0 & $63(54.3)$ & $48(53.3)$ & $26(56.5)$ & $22(50.0)$ \\
\hline $1+$ & $40(34.5)$ & $32(35.6)$ & $16(33.8)$ & $16(36.4)$ \\
\hline $2+$ & $11(9.5)$ & $8(8.9)$ & $4(8.7)$ & $4(9.1)$ \\
\hline Unknown & $2(1.7)$ & $2(2.2)$ & 0 & $2(4.5)$ \\
\hline
\end{tabular}

Data expressed as $n(\%)$ unless otherwise specified. ${ }^{a}$ according to the revised Response Evaluation Criteria in Solid Tumors guideline, version 1.1; AMBC, advanced and metastatic breast cancer; E, endocrine therapy alone; EC, endocrine therapy plus capecitabine; ECOG PS, Eastern Cooperative Oncology Group performance status; ER, estrogen receptor; IHC, immunohistochemistry; mTOR, mammalian target of rapamycin; PgR, progesterone receptor.

Most patients in each group received endocrine therapy with an aromatase inhibitor, or if premenopausal, an aromatase inhibitor plus a luteinizing-hormone-releasing hormone (LHRH) agonist: $28(60.9 \%)$ of the 46 patients in group E and $27(61.4 \%)$ of the 44 patients in group EC (Table 2). A total of 81 of the 116 patients had complete response (CR) or partial response (PR), giving an objective response rate (ORR) of $69.8 \%$ (95\% CI, 60.6-78.0\%). 
The ORRs (combining CR and PR) to the bevacizumab-paclitaxel induction therapy were $76.1 \%(35 / 46)$ and $77.3 \%(34 / 44)$ in groups $\mathrm{E}$ and EC, respectively (Table 3$)$.

Table 2. Endocrine agents used as maintenance therapy.

\begin{tabular}{ccc}
\hline Endocrine Agent(s) & Group E $(\boldsymbol{n}=\mathbf{4 6 )}$ & Group EC $(\boldsymbol{n}=\mathbf{4 4})$ \\
\hline $\mathrm{AI} \pm$ LHRH Agonist & $28(60.9)$ & $27(61.4)$ \\
SERM & $11(23.9)$ & $8(18.2)$ \\
SERD (Fulvestrant) & $6(13.0)$ & $6(13.6)$ \\
MPA & $1(2.2)$ & $3(6.8)$
\end{tabular}

Data expressed as $n(\%)$. AI, aromatase inhibitor; E, endocrine therapy alone; EC, endocrine therapy plus capecitabine; LHRH, luteinizing-hormone-releasing hormone; MPA, medroxyprogesterone acetate; SERD, selective estrogen receptor downregulator; SERM, selective estrogen receptor modulator (tamoxifen, toremifene).

Table 3. Response to bevacizumab-paclitaxel induction therapy.

\begin{tabular}{ccc}
\hline Response & Group E $(\boldsymbol{n}=\mathbf{4 6})$ & Group EC $(\boldsymbol{n}=\mathbf{4 4})$ \\
\hline Complete Response & 0 & $1(2.3)$ \\
Partial Response & $35(76.1)$ & $33(75.0)$ \\
Stable Disease & $11(23.9)$ & $10(22.7)$ \\
Progressive Disease & 0 & 0 \\
Not Evaluable & 0 & 0 \\
\hline
\end{tabular}

Data expressed as $n(\%)$. E, endocrine therapy alone; EC, endocrine therapy plus capecitabine.

At the data cutoff point (September 2018), the median follow-up period was 30.8 months (range, 6.1-72.1 months). By that time, 80 (88.9\%) of the 90 patients had discontinued maintenance therapy $(43 / 46,93.5 \%$, in group $\mathrm{E}$, and $37 / 44,84.1 \%$, in group $\mathrm{EC})$, all due to $\mathrm{PD}$, except for 1 patient in group EC, which was due to AE. A total of $54(67.5 \%)$ of the patients who discontinued maintenance therapy had been switched to bevacizumab-paclitaxel reinduction therapy, and 26 (13 from each group) had been switched to another therapy or breast-conserving surgery (Figure 1).

\subsection{Efficacy}

\subsubsection{PFS of Maintenance Therapy}

The median PFS of maintenance therapy (primary endpoint) was significantly longer in group EC than in group E (11.1 months, 95\% CI 8.0-11.8 months, versus 4.3 months, 95\% CI 3.6-6.0 months; $p<0.01$ ) (Figure 2). The hazard ratio (HR) for PFS was 0.53 (95\% CI, $0.34-0.83)$. There was no significant difference in the ORR between group $\mathrm{E}(32.6 \%)$ and group EC (50.0\%). The disease control rate (DCR) was higher in group EC than in group E (72.7\%, 32/44 patients versus $50.0 \%, 23 / 46$ patients; $p=0.03)$.

As for the ad hoc subgroup analysis, the factors affecting the PFS of maintenance therapy were investigated (Supplementary Figure S1). The addition of capecitabine tended to be beneficial in all subgroups, particularly in patients who had previously received $\geq 3$ regimens of endocrine therapy for AMBC and those in whom induction therapy had produced CR or PR. 


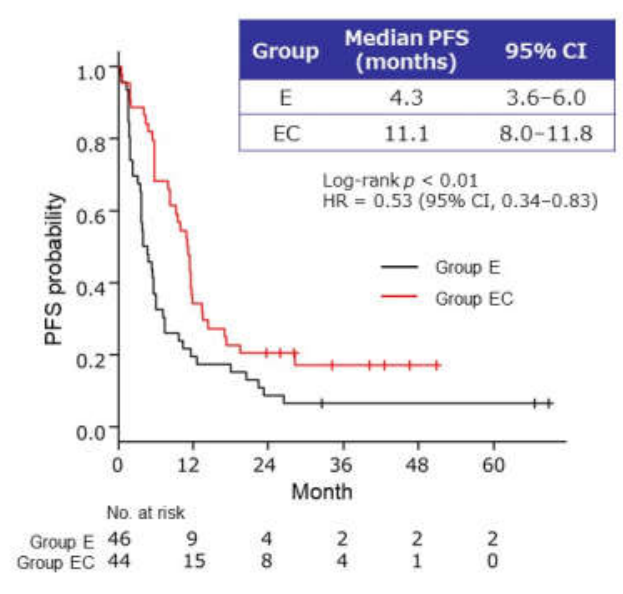

Figure 2. Kaplan-Meier curves of progression-free survival (PFS) in patients who received maintenance therapy with endocrine therapy alone (group E) or endocrine therapy plus capecitabine (group EC) after bevacizumab-paclitaxel induction therapy. CI, confidence interval; E, endocrine therapy alone; EC, endocrine therapy plus capecitabine; HR, hazard ratio.

\subsubsection{Efficacy of Reinduction Therapy}

A total of $54(67.5 \%)$ of the 80 patients who had discontinued maintenance therapy were switched to bevacizumab-paclitaxel reinduction therapy: 30 (65.2\%) of the 46 patients in group E and 24 (54.5\%) of the 44 patients in group EC. The median PFS, ORR, and DCR of bevacizumab-paclitaxel reinduction therapy after either of the two maintenance therapies were 7.8 months (95\% CI, 6.7-9.5 months), 20.4\% (11/54 patients), and 63.0\% (34/54 patients), respectively. Nine patients (16.7\%) did not respond to the reinduction therapy and were found to have PD.

The median PFS of bevacizumab-paclitaxel reinduction therapy tended to be longer in group E than in group EC; however, no significant difference was found between the groups (9.1 months, 95\% CI 6.7-11.3 months, and 7.8 months, 95\% CI 5.5-9.5 months, respectively; $\log$-rank $p=0.053$ ) (Figure 3).

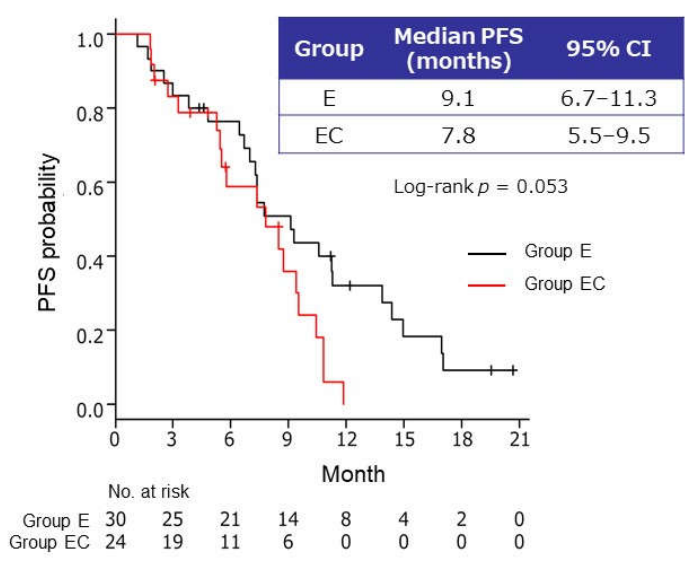

Figure 3. Kaplan-Meier curves of progression-free survival (PFS) in patients who received bevacizumab-paclitaxel reinduction therapy after maintenance therapy with endocrine therapy alone (group E) or endocrine therapy plus capecitabine (group EC). CI, confidence interval; $\mathrm{E}$, endocrine therapy alone; EC, endocrine therapy plus capecitabine.

\subsubsection{Time to Failure of Strategy}

Analysis of data from the 90 patients randomized to maintenance therapy found no significant difference between the two groups in terms of the median time to failure of strategy (13.9 months, 95\% CI 8.7-17.8 months, and 16.6 months, 95\% CI 11.5-19.6 months, in groups E and EC, respectively) (Figure 4). 


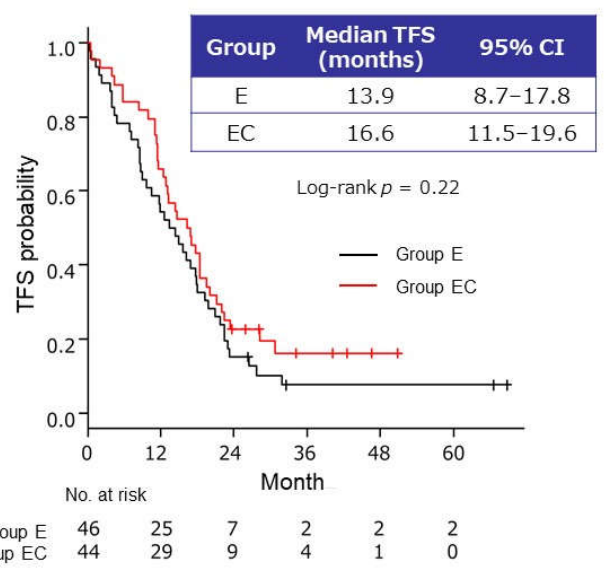

Figure 4. Kaplan-Meier curves of time to failure of strategy (TFS) from randomization in patients who received bevacizumab-paclitaxel reinduction therapy after maintenance therapy with endocrine therapy alone (group E) or endocrine therapy plus capecitabine (group EC). CI, confidence interval; $\mathrm{E}$, endocrine therapy alone; EC, endocrine therapy plus capecitabine.

\subsubsection{OS from the Start of Induction Therapy}

The median OS from the start of bevacizumab-paclitaxel induction therapy was significantly longer in group EC than in group E: 43.8 months (95\% CI, 33.7 months to not estimable, NE, versus 34.9 months (95\% CI 23.3 months to NE)) (HR: $0.41,95 \%$ CI $0.17-0.99$; $\log$-rank $p=0.046)$. In groups EC and E, the OS rates were $83.5 \%(68.5-91.8 \%)$ and $62.3 \%$ $(46.6-74.6 \%)(\log$-rank $p=0.02)$, respectively, at 24 months, and $62.6 \%(44.5-76.3 \%)$ and $47.6 \%$ (32.2-61.5\%), respectively, at 36 months (Figure 5).

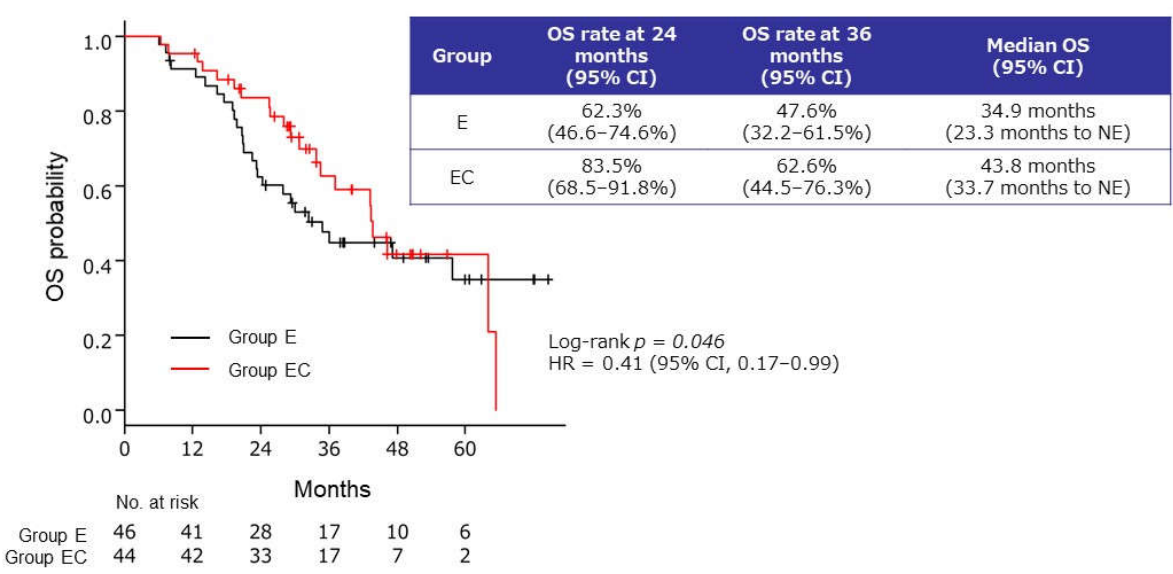

Figure 5. Kaplan-Meier curves of overall survival (OS) rate from the start of bevacizumab-paclitaxel induction therapy in patients who received maintenance therapy with endocrine therapy alone (group E) or endocrine therapy plus capecitabine (group EC). CI, confidence interval; E, endocrine therapy alone; EC, endocrine therapy plus capecitabine; HR, hazard ratio; NE, not estimable.

As for the ad hoc subgroup analysis, the factors affecting OS from the start of induction therapy were investigated (Supplementary Figure S2). The addition of capecitabine to endocrine maintenance therapy benefitted all subgroups; in particular, a significant superiority to endocrine therapy alone was noted in patients who had previously received $\geq 3$ regimens of endocrine therapy for AMBC.

\subsection{Safety}

All 90 patients assigned to maintenance therapy received at least one dose of the study treatment. Therefore, their data were included in the safety analysis set. In groups 
E and EC, 174 AEs (grade $\geq 3$ AEs: 12) and 299 AEs (grade $\geq 3$ AEs: 17) were recorded, respectively. Table 4 lists AEs that were experienced by $\geq 10 \%$ of the patients or grade $\geq 3$. All cases of grade $\geq 3$ AEs were manageable, and no patients discontinued the study treatment due to AEs.

In each group, sensory neuropathy and alopecia were experienced by most patients $(>65 \%)$, and hypertension by more than a third. The incidence of AEs considered by the investigators to be related to capecitabine, namely, palmar-plantar erythrodysesthesia syndrome, fatigue, malaise, anorexia, nail disorders, skin changes, and dysgeusia, was higher in group EC than in group E (affecting $\geq 27.3 \%$ versus $\leq 15.2 \%$ ). However, these AEs were grade $\geq 3$ in only 7 cases ( 6 cases of palmar-plantar erythrodysesthesia syndrome and 1 case of fatigue) in group EC.

Regarding hematologic AEs, the incidence of anemia was similar in both groups: $17.4 \%$ $(8 / 46)$ and $20.5 \%(9 / 44)$ in groups $E$ and EC, respectively. Neutropenia was experienced in $22.7 \%$ in group EC but was grade $\geq 3$ in 1 case.

Regarding serious AEs other than symptoms associated with worsening of disease, there was 1 case of bilateral pulmonary artery thrombus formation during maintenance therapy (outcome: improved) and 1 case of grade 4 intraventricular hemorrhage during bevacizumab-paclitaxel reinduction therapy.

Table 4. Incidence of adverse events (experienced by $\geq 10 \%$ of patients or grade $\geq 3$ in either group) in patients receiving endocrine therapy alone (group E) or endocrine therapy plus capecitabine (group EC) as maintenance therapy after bevacizumab-paclitaxel induction therapy.

\begin{tabular}{|c|c|c|c|c|}
\hline \multirow{2}{*}{ Adverse Event } & \multicolumn{2}{|c|}{ Group E $(n=46)$} & \multicolumn{2}{|c|}{ Group EC $(n=44)$} \\
\hline & $n$ & Grade $\geq 3$ & $n$ & Grade $\geq 3$ \\
\hline Sensory Neuropathy & $31(67.4)$ & $2(4.3)$ & $34(77.3)$ & $2(4.5)$ \\
\hline Alopecia & $30(65.2)$ & $0(0.0)$ & $30(68.2)$ & $0(0.0)$ \\
\hline Hypertension & $16(34.8)$ & $3(6.5)$ & $21(47.7)$ & $5(11.4)$ \\
\hline Hemorrhage & $9(19.6)$ & $0(0.0)$ & $11(25.0)$ & $0(0.0)$ \\
\hline Proteinuria & $9(19.6)$ & $0(0.0)$ & $4(9.1)$ & $1(2.3)$ \\
\hline Anemia & $8(17.4)$ & $0(0.0)$ & $9(20.5)$ & $0(0.0)$ \\
\hline Malaise & $7(15.2)$ & $0(0.0)$ & $14(31.8)$ & $0(0.0)$ \\
\hline Motor Neuropathy & $7(15.2)$ & $0(0.0)$ & $8(18.2)$ & $0(0.0)$ \\
\hline Arthralgia & $7(15.2)$ & $1(2.2)$ & $7(15.9)$ & $0(0.0)$ \\
\hline Anorexia & $6(13.0)$ & $1(2.2)$ & $14(31.8)$ & $0(0.0)$ \\
\hline ALT Increased & $6(13.0)$ & $2(4.3)$ & $1(2.3)$ & $0(0.0)$ \\
\hline Rash, Skin Changes (Dryness/Itchiness) & $5(10.9)$ & $0(0.0)$ & $12(27.3)$ & $0(0.0)$ \\
\hline Limb Edema & $5(10.9)$ & $0(0.0)$ & $9(20.5)$ & $0(0.0)$ \\
\hline Oral Mucositis & $5(10.9)$ & $0(0.0)$ & $8(18.2)$ & $0(0.0)$ \\
\hline Fatigue & $4(8.7)$ & $1(2.2)$ & $17(38.6)$ & $1(2.3)$ \\
\hline Nausea & $4(8.7)$ & $0(0.0)$ & $10(22.7)$ & $0(0.0)$ \\
\hline AST Increased & $4(8.7)$ & $2(4.3)$ & $1(2.3)$ & $0(0.0)$ \\
\hline Dysgeusia & $3(6.5)$ & $0(0.0)$ & $12(27.3)$ & $0(0.0)$ \\
\hline Diarrhea & $3(6.5)$ & $0(0.0)$ & $7(15.9)$ & $0(0.0)$ \\
\hline Muscle Pain & $2(4.3)$ & $0(0.0)$ & $6(13.6)$ & $0(0.0)$ \\
\hline $\begin{array}{l}\text { Palmar-Plantar Erythrodysesthesia } \\
\text { Syndrome }\end{array}$ & $1(2.2)$ & $0(0)$ & $33(75.0)$ & $6(13.6)$ \\
\hline Neutropenia & $1(2.2)$ & $0(0.0)$ & $10(22.7)$ & $1(2.3)$ \\
\hline Leukopenia & $1(2.2)$ & $0(0.0)$ & $7(15.9)$ & $0(0.0)$ \\
\hline Nail Disorders & $0(0.0)$ & $0(0.0)$ & $13(29.5)$ & $0(0.0)$ \\
\hline CPK Increased & $0(0.0)$ & $0(0.0)$ & $1(2.3)$ & $1(2.3)$ \\
\hline
\end{tabular}

Data expressed as $n$ (\%). ALT, alanine aminotransferase; AST, aspartate aminotransferase; CPK, creatine phosphokinase; E, endocrine therapy alone; $\mathrm{EC}$, endocrine therapy plus capecitabine. 


\section{Discussion}

\subsection{Maintenance Therapy: Clinical Usefulness of the Addition of Capecitabine to Endocrine Therapy}

In the present study, we evaluated two maintenance therapies in patients with HRpositive, HER2-negative AMBC to determine the clinical usefulness of the addition of capecitabine to maintenance endocrine therapy, as compared with endocrine therapy alone, in patients who had received bevacizumab-paclitaxel induction therapy. The results showed that the addition of capecitabine extended the median PFS from 4.3 months to 11.1 months, representing a $47 \%$ reduction in risk of disease progression or death. In terms of tolerability and safety, the results showed both maintenance therapies to have manageable and tolerable toxicity. These findings are consistent with the previous literature suggesting that maintenance therapy with capecitabine may be beneficial in terms of PFS with preservation of QOL [20].

Previously, the IMELDA trial evaluated the efficacy of bevacizumab-capecitabine maintenance therapy, after bevacizumab-docetaxel induction therapy, in patients with HER2-negative metastatic breast cancer [16]; the median PFS was significantly longer in patients who received the bevacizumab-capecitabine regimen than in those who received bevacizumab only (11.9 months vs. 4.3 months; HR, 0.38 ), and an even more marked improvement was shown for the median OS (39.0 months vs. 23.7 months; HR: 0.43). In this trial, the docetaxel used for induction therapy was switched to capecitabine for maintenance therapy to avoid the AEs (including neuropathy and edema) that limit the long-term use of taxanes. Additionally, the AROBASE study [21] compared the effects of maintenance bevacizumab-exemestane therapy with those of continuous bevacizumab-taxane induction therapy; the median PFS from randomization was 7.6 (95\% CI, 5.4-10.9) months in patients who were switched to the maintenance bevacizumab-exemestane regimen, and 8.1 (95\% CI, 6.5-10.7) months in patients who continuously received the bevacizumabtaxane regimen. Although the results failed to show the superiority of maintenance therapy, subgroup analyses suggested that patients without a previous history of hormone resistance, especially in the case of treatment naïve to aromatase inhibitor therapy, may benefit from the maintenance strategy. Moreover, the Dutch Breast Cancer Research Group compared intermittent versus continuous first-line treatment with bevacizumab plus paclitaxel; unfortunately, this strategy was not successful to demonstrate the noninferiority of the intermittent regimen to continuous treatment in terms of PFS or OS [22]. Promising results have also been reported in a Japanese phase 2 trial in which patients underwent induction therapy with bevacizumab plus paclitaxel followed by maintenance therapy with eribulin (median PFS: 10.7 months; OS: 20.0 months) [23]. Therefore, previous studies examining maintenance therapy have not yielded consistent results, and thus there is a need to explore a maintenance therapy with an optimal balance of toxicity and efficacy.

The regimen used in the present study, that is, maintenance endocrine therapy with or without the addition of capecitabine, is unique in that bevacizumab was suspended during maintenance therapy. While continuous bevacizumab may be useful to prolong PFS, its long-term use is associated with proteinuria, which often prevents treatment continuation. To resolve this problem, we investigated a treatment strategy that includes bevacizumab-paclitaxel reinduction therapy so that bevacizumab can be suspended during maintenance therapy. The addition of capecitabine to endocrine maintenance therapy resulted in favorable PFS (11.1 months; 95\% CI, 8.0-11.8 months). We consider the finding to be meaningful, because the result of this regimen, even without bevacizumab, was comparable to that reported in the IMELDA trial [16], and better than those reported in the AROBASE study [21].

\subsection{Efficacy of Bevacizumab-Paclitaxel Reinduction Therapy}

In 54 of 80 patients who were successfully switched to bevacizumab-paclitaxel reinduction therapy, PFS was 7.8 months. Added to the 6 months of PFS under induction therapy, the total treatment duration for bevacizumab-paclitaxel was $>1$ year, a similar 
length of time to the PFS achieved in the E2100 [11] and MERiDiAN trials [10,12]. Of the patients who did not receive reinduction therapy, about half chose endocrine therapy or other anticancer therapies. This suggests that they wished to avoid AEs such as peripheral neuropathy and alopecia, which they might have experienced while undergoing induction therapy or the symptom improved during maintenance therapy.

\subsection{Effects on OS from the Start of Induction Therapy}

The addition of capecitabine to endocrine maintenance therapy improved the OS rate (increase in point estimate by $21.2 \%$ and $15 \%$ at 24 and 36 months, respectively) from the start of induction therapy, consistent with the finding in the IMELDA study [16], suggesting that the increased PFS during maintenance therapy could lead to a positive effect on OS. The effect of capecitabine after bevacizumab-paclitaxel induction therapy may be explained by the mechanism discussed in the CREATE- $X$ trial report; taxanes may induce thymidine phosphorylase in cancer cells, which activates capecitabine, resulting in increased antitumor effect $[17,24]$.

The finding inversely suggests that endocrine-only therapy may not be sufficient at the maintenance stage after induction therapy. However, it should be noted that, in the present study, nearly $30 \%$ of the randomized patients had received $\geq 3$ endocrine therapy regimens for $\mathrm{AMBC}$ at baseline. Therefore, in this population of patients, the benefits of maintenance endocrine therapy at this point may be suboptimal, potentially leading to the observed short PFS in group E compared with group EC. This is also supported by the finding from the ad hoc subgroup analysis showing previous use of $\geq 3$ regimens of endocrine therapy as a factor affecting the PFS of maintenance therapy (Supplementary Figure S1) and OS (Supplementary Figure S2) in favor of group EC. Although limited by the small sample size, the results may also be interpreted as follows: the necessity of $\geq 3$ regimens of endocrine therapy may indicate that tumors were more sensitive to endocrine therapy at baseline; however, the sensitivity may be weakened because of the long-term endocrine therapy. Regarding poststudy treatment, we did not collect relevant data (i.e., treatment details after completion of maintenance therapy and after completion of bevacizumab-paclitaxel reinduction therapy) through the electronic data capture system. However, as capecitabine is recommended as a standard treatment for AMBC by the guidelines and it is reimbursed by Japanese health insurance, it is possible that many patients in group $\mathrm{E}$ received treatment with capecitabine upon progression.

Since CDK4/ 6 inhibitors have now become available, the combination of endocrine therapy and a CDK4/ 6 inhibitor may be a possible option as maintenance therapy. Future study will be needed to determine whether capecitabine or a CDK4/6 inhibitor is more appropriate to be added to maintenance endocrine therapy in terms of prolonging OS.

\subsection{Implications for Therapeutic Strategy}

With the introduction of CDK4/ 6 inhibitors, endocrine therapy plus a CDK4/ 6 inhibitor is used as standard first- or second-line therapy for HR-positive, HER2-negative AMBC; it has demonstrated clinical benefit in terms of improved PFS with less toxicity as compared with a chemotherapy regimen [25-27]. However, the meta-analysis of data from the MONALEESA trials found differences in the efficacy of concomitant CDK4/6 inhibitors when patients with HR-positive, HER2-negative AMBC were stratified by a PAM50-based subtype [28]. For example, patients with the basal-like subtype are unlikely to benefit from the use of CDK4/ 6 inhibitors. For these patients, as well as those who had progression after treatment with CDK4/ 6 inhibitors, chemotherapies should be considered at an early treatment phase to eliminate the highly malignant tumors, and then followed by maintenance therapy that is less toxic. This concept was suggested by the IMELDA trial [16], and supported by the present study findings, in which chemotherapy-containing maintenance therapy was beneficial in terms of both PFS and OS. 


\subsection{Limitations and Future Research}

The present phase 2 trial was not designed to compare different combinations of endocrine agents and capecitabine. Additionally, it was not designed to investigate whether alternatives to capecitabine, such as CDK4/6 inhibitors, would be efficacious in combination with endocrine maintenance therapy, or whether bevacizumab should be added to maintenance therapy. It was aimed at determining which of the maintenance therapies (endocrine therapy alone or capecitabine plus endocrine therapy) should move on to phase 3 , which will then compare the standard treatment (continuing initial chemotherapy until reaching $\mathrm{PD}$ ) and chemotherapy followed by maintenance therapy.

As CDK4/6 inhibitors had not been approved in Japan during the present study period, no patients treated with CDK4/6 inhibitors were included. The combination of endocrine therapy and a CDK4/6 inhibitor represents the recent standard first- and second-line treatment. However, as there has been no evidence suggesting that the use of a CDK4/ 6 inhibitor affects the sensitivity to subsequent chemotherapy, we believe that data from the present study evaluating the usefulness of adding capecitabine to endocrine therapy can also be extrapolated to patients previously treated with CDK4/6 inhibitors, given that they are still in need of chemotherapy.

About one-third of the patients ( $n=13$ each) in groups E and EC did not receive reinduction therapy. This may have affected the OS, given that the PFS of the reinduction therapy tended to be longer in group E than in group EC, as opposed to the result of the PFS of maintenance therapy.

As this was a phase 2 study, a limited number of patients were enrolled. However, patients were randomly assigned into the groups so as to minimize the effects of confounding factors. Moreover, the results of the subgroup analyses showed straightforward results; consistent superiority of group EC in terms of PFS (Supplementary Figure S1) and OS (Supplementary Figure S2) was shown. Therefore, we consider that discrepancies between the groups unlikely affected the present study findings. A meta-analysis of data and a phase 3 study are needed to confirm the efficacy of the treatment strategy comprising induction, maintenance, and reinduction therapy in AMBC patients and establish optimal therapeutic strategies. Additionally, translational research analysis of serum samples collected at each evaluation point is still ongoing; the results will be presented in due course.

\section{Materials and Methods}

\subsection{Study Design}

The present study, KBCSG-TR1214 (UMIN Clinical Trials Registry trial no. UMIN000008662), was a multicenter, parallel-group, open-label, randomized phase 2 trial for HR-positive, HER2-negative patients who had undergone no or one prior chemotherapy for AMBC. The study design is shown in Figure 6.

\subsection{Recruitment}

Patients were recruited from hospitals affiliated with Kinki Breast Cancer Study Group-Translational Research in Japan and registered at the Data Coordinating Center, Osaka University Hospital, Osaka, Japan. Eligible patients were women aged $\geq 20$ years with advanced breast cancer with distant metastases or inoperable recurrent breast cancer who were expected to benefit from endocrine therapy. The other key inclusion criteria at primary registration were estrogen-receptor-positive status confirmed immunohistochemically, HER2-negative status confirmed by fluorescence in situ hybridization or immunohistochemically, Eastern Cooperative Oncology Group performance status of 0 or 1 , life expectancy $\geq 12$ months, evaluable lesion (according to the revised Response Evaluation Criteria in Solid Tumors (RECIST) guideline, version 1.1), previous treatment with no more than one chemotherapy regimen for metastatic or inoperable recurrent breast cancer, no previous treatment with paclitaxel (including nab-paclitaxel), oral fluoropyrimidines (with the exception of administration in a neoadjuvant or adjuvant setting, or $\geq 12$ months 
before primary registration) or bevacizumab, and adequate organ function (confirmed by laboratory testing within 2 weeks before receiving the first dose of any study drug).

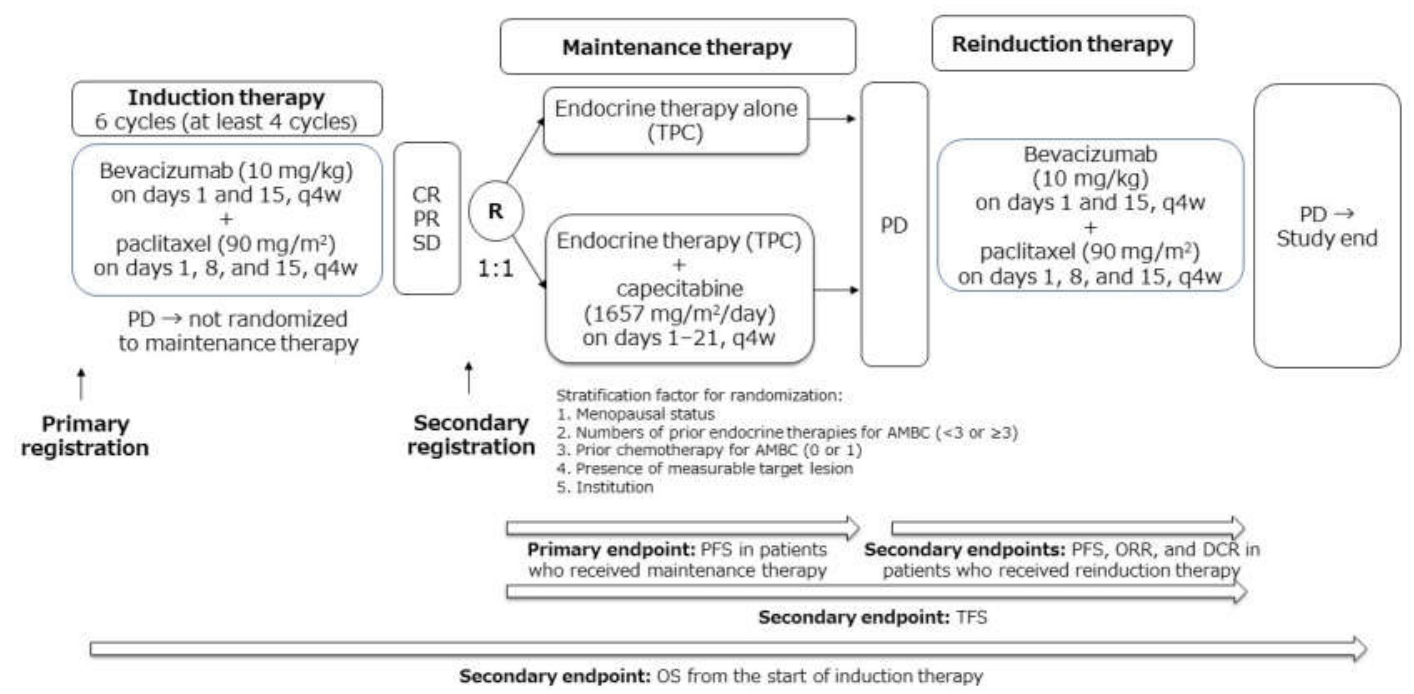

Figure 6. Study design. AMBC, advanced and metastatic breast cancer; DCR, disease control rate; CR, complete response; ORR, objective response rate; $\mathrm{PD}$, progressive disease; PFS, progression-free survival; PR, partial response; R, randomization; $\mathrm{SD}$, stable disease; TFS, time from randomization to failure of strategy; TPC, treatment of physician's choice; q4w, every 4 weeks.

The key exclusion criteria at primary registration included brain metastases, pregnancy, and ineligibility based on the decision of the attending physician or the principal investigator.

The key inclusion criteria at secondary registration included CR, PR, or stable disease (SD), confirmed by imaging at the end of cycle 6 of induction therapy; CR, PR, or SD (confirmed by imaging) in case of discontinuation after $\geq 4$ cycles of induction therapy due to AEs; no obvious sign of PD (confirmed by imaging at the end of induction therapy), and no clinical worsening, in patients with a small lesion (maximum diameter, $<10 \mathrm{~mm}$, or minimum lymph node diameter, $\geq 10 \mathrm{~mm}$ and $<15 \mathrm{~mm}$ ) only, bone lesions only, pleural lesions only, or diffuse skin lesions only (this criterion also applied to patients who received $\geq 4$ cycles of induction therapy but discontinued due to AEs); and adequate organ function (confirmed by laboratory testing).

\subsection{Treatment}

\subsubsection{Induction Therapy}

The initial treatment was induction therapy comprising 6 cycles of bevacizumab and paclitaxel. Each cycle was 28 days. Patients received bevacizumab $(10 \mathrm{mg} / \mathrm{kg}$ on days 1 and 15) and paclitaxel (90 mg/ $\mathrm{m}^{2}$ on days 1,8 , and 15). During induction therapy, concomitant use of other anticancer therapies, or of antithrombotic agents (other than aspirin $\leq 324 \mathrm{mg}$ /day), was prohibited.

\subsubsection{Randomization and Maintenance Therapy}

After 6 cycles ( $\geq 4$ cycles in case of discontinuation due to AEs) of induction therapy, patients without $\mathrm{PD}$ were randomly assigned in a 1:1 ratio to receive maintenance therapy with either endocrine therapy alone (group E) or endocrine therapy plus capecitabine (group EC). The stratification factors used at randomization were menopausal status, presence of a measurable lesion, institution, number of prior endocrine therapies for AMBC $(<3$ or $\geq 3)$, and whether or not the treatment was first-line chemotherapy for AMBC.

In both group $\mathrm{E}$ and group $\mathrm{EC}$, the choice of endocrine agents was at the discretion of the attending physicians. The drugs were administered in accordance with the package inserts. In group EC, patients additionally received capecitabine, $1657 \mathrm{mg} / \mathrm{m}^{2} /$ day 
twice daily on days 1-21, followed by a 7-day rest period. This metronomic regimen of capecitabine was based on previous studies indicating a less toxic profile associated with low-dose capecitabine $[29,30]$. Prohibited treatments during maintenance therapy were the same as those during induction therapy. Use of LHRH agonists (goserelin and leuprorelin) was permitted during maintenance therapy.

\subsubsection{Reinduction Therapy}

In cases of PD despite maintenance therapy, patients were switched to bevacizumabpaclitaxel reinduction therapy. In cases of suspension or discontinuation of either drug (i.e., bevacizumab or paclitaxel), administration of the other continued. If neither drug could be administered within $28(+3)$ days of the most recent dose, the reinduction therapy was judged to be terminated.

\subsection{Assessment of Efficacy and Safety}

Efficacy was assessed according to the revised RECIST guideline, version 1.1 [31]. Adverse events were defined as any unfavorable or unintended sign that may or may not be associated with the study treatment. Periodic monitoring was carried out, and all AEs were recorded and graded in accordance with the National Cancer Institute Common Terminology Criteria for Adverse Events, version 4.0 (Japanese Clinical Oncology Group edition) [32].

Follow-up was for 3 years after completion of registration, or for patients registered after August 2015, the end of treatment.

\subsection{Endpoints}

The primary endpoint was PFS of maintenance therapy (defined as the period from the secondary registration to the first of either PD or death from any cause). The main secondary endpoints were time to failure of strategy (defined as the period from the secondary registration to the first of either PD during the reinduction therapy, death from any cause, or date of treatment discontinuation-in the case of patients who did not receive reinduction therapy, date of PD during maintenance therapy); ORR (defined as proportion of patients with CR or PR) and DCR (defined as proportion of patients with CR, PR, or SD) of reintroduction therapy; PFS of reinduction therapy (defined as the period from the start of reinduction therapy to the first of either PD or death from any cause) and time to treatment failure of reintroduction therapy (defined as the period from the start of reinduction therapy to the first of either PD, death from any cause, or date of treatment discontinuation); OS (defined as the period from the primary registration to death from any cause); and safety.

\subsection{Statistical Analyses}

Sample size calculations were calculated based on a type 1 error (one sided) of 0.05 , an $80 \%$ power to estimate the median PFS in patients who had received maintenance therapy (endocrine therapy with or without capecitabine) for 9 months (threshold 6 months), a 2-year enrollment period, and a 1-year follow-up period, and accounted for the exclusion of ineligible patients. The target number of patients enrolled and the target number randomized after induction therapy (having completed primary registration) were 120 and 90, respectively.

Data used in the efficacy analysis were from the full analysis set, defined as patients who fulfilled the inclusion criteria and were enrolled to receive study treatment. The safety analysis used data from all patients who received at least one dose of any of the maintenance therapy drugs. The Kaplan-Meier method was used to generate curves for PFS and other survival endpoints, and to calculate related statistics, for groups E and EC.

The trial was designed not to directly compare the results for groups E and EC but rather to determine whether the survival endpoint results for each group met prespecified criteria: threshold median PFS $\geq 6.0$ months and expected median PFS $\geq 9$ months. 
By means of comprehensive assessment of the results for these and other endpoints (including incidence of AEs), we aimed to determine which therapies should move on to phase 3. Both groups E and EC were experimental arms. Therefore, the following comparisons were made for the purpose of reference only. HRs and their 95\% CIs were calculated by Cox regression analysis, and intergroup comparisons were performed by the log-rank test. Based on the Cox model, ad hoc subgroup analyses using stratification factors at randomization were carried out to identify factors affecting survival. Regarding the response rate (ORR and DCR), Fisher's exact test was used to compare the results between groups E and EC. Statistical analyses were carried out using SPSS Statistics 26.0 (IBM Corp., Armonk, NY, USA) and R (version 3.5.2) [33].

\section{Conclusions}

We found that bevacizumab-paclitaxel induction therapy followed by maintenance endocrine therapy and subsequent bevacizumab-paclitaxel reinduction therapy could be a new therapeutic strategy for HR-positive, HER2-negative AMBC. Our results suggest that the addition of capecitabine to endocrine therapy may be more beneficial than endocrine therapy alone as maintenance therapy.

Supplementary Materials: The following are available online at https: / www.mdpi.com/article/ 10.3390 / cancers13174399/s1, Figure S1: Forest plot for progression-free survival (PFS, months) in patients who received maintenance therapy with endocrine therapy alone (E) or endocrine therapy plus capecitabine (EC), Figure S2: Forest plot for overall survival (OS, months) from the start of bevacizumab-paclitaxel induction therapy in patients who received maintenance therapy with endocrine therapy alone (group E) or endocrine therapy plus capecitabine (group EC).

Author Contributions: Each author's contributions to the manuscript are as follows: conceptualization, N.M., T.Y. (Tetsuhiro Yoshinami), and T.N.; study design, N.M., T.Y. (Tetsuhiro Yoshinami), Y.K., T.T., M.I. (Mitsuhiko Iwamoto), S.M., and T.N.; data acquisition, N.M., T.Y. (Tetsuhiro Yoshinami), M.M., M.Y., Y.K., T.T., K.Y., J.T., F.F., H.Y., J.Y., H.M., F.A., and T.N.; data analysis, T.Y. (Tomomi Yamada) and S.M.; data interpretation, N.M., T.Y. (Tetsuhiro Yoshinami), M.I. (Masahiko Ikeda), Y.K., J.T., T.Y. (Tomomi Yamada), S.M., and T.N.; writing—original draft preparation, N.M., T.Y. (Tetsuhiro Yoshinami), and T.N.; writing—review and editing, N.M., T.Y. (Tetsuhiro Yoshinami), M.I. (Masahiko Ikeda), M.M., M.Y., Y.K., T.T., K.Y., J.T., M.I. (Mitsuhiko Iwamoto), F.F., H.Y., J.Y., H.M., F.A., T.Y. (Tomomi Yamada), S.M., and T.N. All authors have read and agreed to the published version of the manuscript.

Funding: This study was sponsored by KBCSG-TR and funded by the Supporting Center for Clinical Research and Education, with donations from several pharmaceutical companies, including Chugai Pharmaceutical Co., Ltd.

Institutional Review Board Statement: The study was conducted in compliance with the Declaration of Helsinki and the Ethical Guidelines for Clinical Research of the Ministry of Health, Labour, and Welfare of Japan. Approval from the Institutional Review Board was obtained at each participating institution, including the National Hospital Organization Osaka National Hospital (approval no. 12024, 30 July 2012).

Informed Consent Statement: All patients provided written informed consent at primary registration.

Data Availability Statement: Data are available on request due to privacy/ethical restrictions.

Conflicts of Interest: N.M. has received honorarium (e.g., lecture fees) from Chugai Pharmaceutical, AstraZeneca, Pfizer, Eli Lilly, Eisai, and Takeda Pharmaceutical, and research funding to institution from Chugai Pharmaceutical, AstraZeneca, Kyowa Kirin, MSD, Novartis Pharma, Pfizer, Eli Lilly, Eisai, Sanofi, Nippon Kayaku, and Daiichi Sankyo. T.Y. (Tetsuhiro Yoshinami) has received consulting fee or honorarium from Chugai Pharmaceutical, Pfizer, Novartis Pharma, and AstraZeneca. M.I. (Masahiko Ikeda) has received honorarium from Chugai Pharmaceutical, Eli Lilly, Pfizer, Kyowa Kirin, Novartis Pharma, Bayer Yakuhin, and Eisai. T.T. has received honorarium from Chugai Pharmaceutical. S.M. has received consulting fee or honorarium from AstraZeneca, Bristol Myers Squibb, Chugai Pharmaceutical, Eisai, Eli Lilly, MSD, Pfizer, and Taiho Pharmaceutical. T.N. has received honorarium from Chugai Pharmaceutical, Novartis Pharma, Eli Lilly, Pfizer, Daiichi Sankyo, 
AstraZeneca, and Taiho Pharmaceutical. Other authors have no conflicts of interest to declare related to this study.

\section{References}

1. Hortobagyi, G.N. Treatment of breast cancer. N. Engl. J. Med. 1998, 339, 974-984. [CrossRef] [PubMed]

2. Finn, R.S.; Martin, M.; Rugo, H.S.; Jones, S.; Im, S.A.; Gelmon, K.; Harbeck, N.; Lipatov, O.N.; Walshe, J.M.; Moulder, S.; et al. Palbociclib and Letrozole in Advanced Breast Cancer. N. Engl. J. Med. 2016, 375, 1925-1936. [CrossRef]

3. Turner, N.C.; Ro, J.; André, F.; Loi, S.; Verma, S.; Iwata, H.; Harbeck, N.; Loibl, S.; Huang Bartlett, C.; Zhang, K.; et al. PALOMA3 Study Group. Palbociclib in Hormone-Receptor-Positive Advanced Breast Cancer. N. Engl. J. Med. 2015, 373, 209-219. [CrossRef]

4. Sledge, G.W., Jr.; Toi, M.; Neven, P.; Sohn, J.; Inoue, K.; Pivot, X.; Burdaeva, O.; Okera, M.; Masuda, N.; Kaufman, P.A.; et al. MONARCH 2: Abemaciclib in Combination With Fulvestrant in Women With HR+/HER2- Advanced Breast Cancer Who Had Progressed While Receiving Endocrine Therapy. J. Clin. Oncol. 2017, 35, 2875-2884. [CrossRef] [PubMed]

5. Goetz, M.P.; Toi, M.; Campone, M.; Sohn, J.; Paluch-Shimon, S.; Huober, J.; Park, I.H.; Trédan, O.; Chen, S.C.; Manso, L.; et al. MONARCH 3: Abemaciclib As Initial Therapy for Advanced Breast Cancer. J. Clin. Oncol. 2017, 35, 3638-3646. [CrossRef]

6. Slamon, D.J.; Neven, P.; Chia, S.; Fasching, P.A.; De Laurentiis, M.; Im, S.A.; Petrakova, K.; Bianchi, G.V.; Esteva, F.J.; Martín, M.; et al. Phase III Randomized Study of Ribociclib and Fulvestrant in Hormone Receptor-Positive, Human Epidermal Growth Factor Receptor 2-Negative Advanced Breast Cancer: MONALEESA-3. J. Clin. Oncol. 2018, 36, 2465-2472. [CrossRef]

7. Tripathy, D.; Im, S.A.; Colleoni, M.; Franke, F.; Bardia, A.; Harbeck, N.; Hurvitz, S.A.; Chow, L.; Sohn, J.; Lee, K.S.; et al. Ribociclib plus endocrine therapy for premenopausal women with hormone-receptor-positive, advanced breast cancer (MONALEESA-7): A randomised phase 3 trial. Lancet Oncol. 2018, 19, 904-915. [CrossRef]

8. Miles, D.W.; Chan, A.; Dirix, L.Y.; Cortés, J.; Pivot, X.; Tomczak, P.; Delozier, T.; Sohn, J.H.; Provencher, L.; Puglisi, F.; et al. Phase III study of bevacizumab plus docetaxel compared with placebo plus docetaxel for the first-line treatment of human epidermal growth factor receptor 2-negative metastatic breast cancer. J. Clin. Oncol. 2010, 28, 3239-3247. [CrossRef]

9. Miles, D.W.; de Haas, S.L.; Dirix, L.Y.; Romieu, G.; Chan, A.; Pivot, X.; Tomczak, P.; Provencher, L.; Cortés, J.; Delmar, P.R.; et al. Biomarker results from the AVADO phase 3 trial of first-line bevacizumab plus docetaxel for HER2-negative metastatic breast cancer. Br. J. Cancer 2013, 108, 1052-1060. [CrossRef] [PubMed]

10. Miles, D.; Cameron, D.; Bondarenko, I.; Manzyuk, L.; Alcedo, J.C.; Lopez, R.I.; Im, S.A.; Canon, J.L.; Shparyk, Y.; Yardley, D.A.; et al. Bevacizumab plus paclitaxel versus placebo plus paclitaxel as first-line therapy for HER2-negative metastatic breast cancer (MERiDiAN): A double-blind placebo-controlled randomised phase III trial with prospective biomarker evaluation. Eur. J. Cancer 2017, 70, 146-155. [CrossRef]

11. Miller, K.; Wang, M.; Gralow, J.; Dickler, M.; Cobleigh, M.; Perez, E.A.; Shenkier, T.; Cella, D.; Davidson, N.E. Paclitaxel plus bevacizumab versus paclitaxel alone for metastatic breast cancer. N. Engl. J. Med. 2007, 357, 2666-2676. [CrossRef]

12. Masuda, N.; Takahashi, M.; Nakagami, K.; Okumura, Y.; Nakayama, T.; Sato, N.; Kanatani, K.; Tajima, K.; Kashiwaba, M. First-line bevacizumab plus paclitaxel in Japanese patients with HER2-negative metastatic breast cancer: Subgroup results from the randomized Phase III MERiDiAN trial. Jpn. J. Clin. Oncol. 2017, 47, 385-392. [CrossRef]

13. Gennari, A.; Stockler, M.; Puntoni, M.; Sormani, M.; Nanni, O.; Amadori, D.; Wilcken, N.; D’Amico, M.; DeCensi, A.; Bruzzi, P. Duration of chemotherapy for metastatic breast cancer: A systematic review and meta-analysis of randomized clinical trials. J. Clin. Oncol. 2011, 29, 2144-2149. [CrossRef]

14. Thomssen, C.; Lüftner, D.; Untch, M.; Haidinger, R.; Würstlein, R.; Harbeck, N.; Augustin, D.; Briest, S.; Ettl, J.; Fasching, P.A.; et al. International Consensus Conference for Advanced Breast Cancer, Lisbon 2019: ABC5 Consensus-Assessment by a German Group of Experts. Breast Care 2020, 15, 82-95. [CrossRef]

15. Cardoso, F.; Paluch-Shimon, S.; Senkus, E.; Curigliano, G.; Aapro, M.S.; André, F.; Barrios, C.H.; Bergh, J.; Bhattacharyya, G.S.; Biganzoli, L.; et al. 5th ESO-ESMO international consensus guidelines for advanced breast cancer (ABC 5). Ann. Oncol. 2020, 31, 1623-1649. [CrossRef] [PubMed]

16. Gligorov, J.; Doval, D.; Bines, J.; Alba, E.; Cortes, P.; Pierga, J.Y.; Gupta, V.; Costa, R.; Srock, S.; de Ducla, S.; et al. Maintenance capecitabine and bevacizumab versus bevacizumab alone after initial first-line bevacizumab and docetaxel for patients with HER2-negative metastatic breast cancer (IMELDA): A randomised, open-label, phase 3 trial. Lancet Oncol. 2014, 15, 1351-1360. [CrossRef]

17. Masuda, N.; Lee, S.J.; Ohtani, S.; Im, Y.H.; Lee, E.S.; Yokota, I.; Kuroi, K.; Im, S.A.; Park, B.W.; Kim, S.B.; et al. Adjuvant Capecitabine for Breast Cancer after Preoperative Chemotherapy. N. Engl. J. Med. 2017, 376, 2147-2159. [CrossRef]

18. Ciruelos, E.; Pérez-García, J.M.; Gavilá, J.; Rodríguez, A.; de la Haba-Rodriguez, J. Maintenance Therapy in HER2-Negative Metastatic Breast Cancer: A New Approach for an Old Concept. Clin. Drug Investig. 2019, 39, 595-606. [CrossRef] [PubMed]

19. Yoshinami, T.; Yagi, T.; Okuno, J.; Kittaka, N.; Ishitobi, M.; Sugimoto, N.; Nakayama, T.; Tamaki, Y.; Imamura, F. Efficacy and safety of re-induction therapy with bevacizumab and paclitaxel for metastatic breast cancer. Breast Cancer 2017, 24, 147-151. [CrossRef]

20. Rossi, S.; Schinzari, G.; Basso, M.; Strippoli, A.; Dadduzio, V.; D'Argento, E.; Cassano, A.; Barone, C. Maintenance hormonal and chemotherapy treatment in metastatic breast cancer: A systematic review. Future Oncol. 2016, 12, 1299-1307. [CrossRef] 
21. Trédan, O.; Follana, P.; Moullet, I.; Cropet, C.; Trager-Maury, S.; Dauba, J.; Lavau-Denes, S.; Diéras, V.; Béal-Ardisson, D.; Gouttebel, M.; et al. A phase III trial of exemestane plus bevacizumab maintenance therapy in patients with metastatic breast cancer after first-line taxane and bevacizumab: A GINECO group study. Ann. Oncol. 2016, 27, 1020-1029. [CrossRef] [PubMed]

22. Claessens, A.K.M.; Bos, M.E.M.M.; Lopez-Yurda, M.; Bouma, J.M.; Rademaker-Lakhai, J.M.; Honkoop, A.H.; de Graaf, H.; van Druten, E.; van Warmerdam, L.J.C.; van der Sangen, M.J.C.; et al. Intermittent versus continuous first-line treatment for HER2-negative metastatic breast cancer: The Stop \& Go study of the Dutch Breast Cancer Research Group (BOOG). Breast Cancer Res. Treat. 2018, 172, 413-423. [PubMed]

23. Inoue, K.; Ninomiya, J.; Saito, T.; Kimizuka, K.; Kurosumi, M. Induction therapy with paclitaxel and bevacizumab followed by switch maintenance therapy with eribulin in Japanese patients with HER2-negative metastatic breast cancer: A multicenter, collaborative, open-label, phase II clinical study for the SBCCSG 35 investigators. BMC Cancer 2018, 18, 671.

24. Toi, M.; Atiqur Rahman, M.; Bando, H.; Chow, L.W. Thymidine phosphorylase (platelet-derived endothelial-cell growth factor) in cancer biology and treatment. Lancet Oncol. 2005, 6, 158-166. [CrossRef]

25. Giuliano, M.; Schettini, F.; Rognoni, C.; Milani, M.; Jerusalem, G.; Bachelot, T.; de Laurentiis, M.; Thomas, G.; de Placido, P.; Arpino, G.; et al. Endocrine treatment versus chemotherapy in postmenopausal women with hormone receptor-positive, HER2-negative, metastatic breast cancer: A systematic review and network meta-analysis. Lancet Oncol. 2019, 20, 1360-1369. [CrossRef]

26. Park, Y.H.; Kim, T.Y.; Kim, G.M.; Kang, S.Y.; Park, I.H.; Kim, J.H.; Lee, K.E.; Ahn, H.K.; Lee, M.H.; Kim, H.J.; et al. Palbociclib plus exemestane with gonadotropin-releasing hormone agonist versus capecitabine in premenopausal women with hormone receptor-positive, HER2-negative metastatic breast cancer (KCSG-BR15-10): A multicentre, open-label, randomised, phase 2 trial. Lancet Oncol. 2019, 20, 1750-1759. [CrossRef]

27. Martin, M.; Zielinski, C.; Ruiz-Borrego, M.; Carrasco, E.; Turner, N.; Ciruelos, E.M.; Muñoz, M.; Bermejo, B.; Margeli, M.; Anton, A.; et al. Palbociclib in combination with endocrine therapy versus capecitabine in hormonal receptor-positive, human epidermal growth factor 2-negative, aromatase inhibitor-resistant metastatic breast cancer: A phase III randomised controlled trial-PEARL. Ann. Oncol. 2021, 32, 488-499. [CrossRef] [PubMed]

28. Prat, A.; Chaudhury, A.; Solovieff, N.; Paré, L.; Martinez, D.; Chic, N.; Martínez-Sáez, O.; Brasó-Maristany, F.; Lteif, A.; Taran, T.; et al. Correlative Biomarker Analysis of Intrinsic Subtypes and Efficacy Across the MONALEESA Phase III Studies. J. Clin. Oncol. 2021, 39, 1458-1467. [CrossRef]

29. Watanabe, T.; Katsumata, N.; Sasaki, Y.; Saeki, T.; Aogi, K.; Toi, M.; Holdener, E. A multicenter phase II trial of XelodaTM (Capecitabine) in patients with docetaxel-refractory advanced/metastatic breast cancer. Proc. Am. Soc. Clin. Oncol. 2001, 20, 61b (abstr 1991).

30. Ishida, T.; Kiba, T.; Takeda, M.; Matsuyama, K.; Teramukai, S.; Ishiwata, R.; Masuda, N.; Takatsuka, Y.; Noguchi, S.; Ishioka, C.; et al. Phase II study of capecitabine and trastuzumab combination chemotherapy in patients with HER2 overexpressing metastatic breast cancers resistant to both anthracyclines and taxanes. Cancer Chemother. Pharmacol. 2009, 64, 361-369. [CrossRef]

31. Eisenhauer, E.A.; Therasse, P.; Bogaerts, J.; Schwartz, L.H.; Sargent, D.; Ford, R.; Dancey, J.; Arbuck, S.; Gwyther, S.; Mooney, M.; et al. New response evaluation criteria in solid tumours: Revised RECIST guideline (version 1.1). Eur. J. Cancer 2009, 45, $228-247$. [CrossRef] [PubMed]

32. National Cancer Institute Common Terminology Criteria for Adverse Events, Version 4.0 (Japanese Clinical Oncology Group Edition). Available online: http://www.jcog.jp/doctor/tool/CTCAEv4J_20170310_miekeshi.pdf (accessed on 1 May 2021).

33. R Core Team. R: A Language and Environment for Statistical Computing; R Foundation for Statistical Computing: Vienna, Austria, 2018; Available online: https: / / www.R-project.org (accessed on 1 May 2021). 